\title{
The Top 100 Most-Cited Articles on Nail Psoriasis: A Bibliometric Analysis
}

\author{
Shilpa Malik, MS; Justin T. Matushansky; Charlene Thomas, MS; Shari R. Lipner, MD, PhD
}

To the Editor:

Nail psoriasis is highly prevalent in patients with cutaneous psoriasis and also may present as an isolated finding. There is a strong association between nail psoriasis and development of psoriatic arthritis (PsA). However, publications on nail psoriasis are sparse compared with articles describing cutaneous psoriasis. ${ }^{1}$ Our objectives were to analyze the nail psoriasis literature for content, citations, and media attention.

The Web of Science database was searched for the term nail psoriasis on April 27, 2020, and publications by year, subject, and article type were compiled. Total and average yearly citations were calculated to create a list of the top 100 most-cited articles. First and last authors, sex, and Altmetric Attention Scores were then recorded. The Wilcoxon rank sum test was calculated to compare the relationship of Altmetric scores between nail psoriasis-specific references and others on the list.

In our data set, the average total number of citations was 134.09 (range, 42-1617), with average yearly citations ranging from 2 to 108. Altmetric scores-measures of media attention of scholarly work -were available for 58 of 100 papers (58\%), with an average score of 33.2 (range, 1-509).

Of the top 100 most-cited articles using the search term nail psoriasis, only $20 \%$ focused on nail psoriasis, with the remainder concentrating on psoriasis/PsA. Only $32 \%$ and $24 \%$ of first and last authors, respectively, were female. Fifty-two percent and 31\% of the articles were published in dermatology and arthritis/rheumatology journals, respectively. There was no statistically significant difference in Altmetric scores between nail psoriasis-specific and other articles in our data set $(P=.7551)$.

For the nail psoriasis-specific articles, all 20 highlighted a lack of nail clinical trials, a positive association with PsA, and a correlation of increased cutaneous psoriasis body surface area with increased onychodystrophy likelihood. ${ }^{2}$ Three of $20(15 \%)$ articles stated that nail psoriasis often is overlooked, despite the negative impact on quality of life, ${ }^{1}$ and emphasized the importance of patient compliance owing to the chronic nature of the disease. Only 1 of $20(5 \%)$ articles focused on nail psoriasis treatments. ${ }^{3}$ There was no overlap between the 100 most-cited psoriasis articles from 1970 to 2012 and our top 100 articles on nail psoriasis. ${ }^{4}$

Treatment recommendations for nail psoriasis by consensus were published by a nail expert group in $2019 .{ }^{5}$ For 3 or fewer nails involved, suggested first-line treatment is intralesional matrix injections with triamcinolone acetonide. For more than 3 affected nails, systemic treatment with oral or biologic therapy is recommended. ${ }^{5}$ Although this article is likely to change clinical practice, it did not qualify for our list because it did not garner sufficient citations in the brief period between its publication date and our search (July 2019-April 2020).

This study is subject to several limitations. Only the Web of Science database was utilized, and only the term nail psoriasis was searched, potentially excluding relevant articles. Using total citations biases toward older articles.

Our bibliometric analysis highlights a lack of publications on nail psoriasis, with most articles focusing on psoriasis and PsA. This deficiency in highly cited nail psoriasis references is likely to be a barrier to physicians in managing patients with nail disease. There is a need for controlled clinical trials and better mechanisms to disseminate information on management of nail psoriasis to practicing physicians.

\section{REFERENCES}

1. Williamson L, Dalbeth N, Dockerty JL, et al. Extended report: nail disease in psoriatic arthritis-clinically important, potentially treatable and often overlooked. Rheumatology (Oxford). 2004;43:790-794. doi:10.1093 /rheumatology/keh198

2. Reich K. Approach to managing patients with nail psoriasis. J Eur Acad Dermatol Venereol. 2009;23(suppl 1):15-21. doi:10.1111 /j.1468-3083.2009.03364.x

3. de Berker D. Management of nail psoriasis. Clin Exp Dermatol. 2000;25:357-362. doi:10.1046/j.1365-2230.2000.00663.x

4. Wu JJ, Choi YM, Marczynski W. The 100 most cited psoriasis articles in clinical dermatologic journals, 1970 to 2012. J Clin Aesthet Dermatol. 2014;7:10-19.

5. Rigopoulos D, Baran R, Chiheb S, et al. Recommendations for the definition, evaluation, and treatment of nail psoriasis in adult patients with no or mild skin psoriasis: a dermatologist and nail expert group consensus. J Am Acad Dermatol. 2019;81:228-240. doi:10.1016/j.jaad.2019.01.072

Ms. Malik is from Touro College of Osteopathic Medicine, Middletown, New York. Mr. Matushansky and Dr. Lipner are from the Department of Dermatology, Weill Cornell Medicine, New York, New York. Ms. Thomas is from the Division of Biostatistics and Epidemiology, Weill Cornell Medicine. The authors report no conflict of interest.

The eTable is available in the Appendix online at www.mdedge.com/dermatology.

Correspondence: Shari R. Lipner, MD, PhD, 1305 York Ave, 9th Floor, New York, NY 10021 (shl9032@med.cornell.edu). doi:10.12788/cutis.0311 


\section{APPENDIX}

eTABLE. Top 100 Most-Cited Articles on Nail Psoriasis From the Web of Science Database

\begin{tabular}{|c|c|c|c|}
\hline Reference & $\begin{array}{l}\text { Total } \\
\text { citations }\end{array}$ & $\begin{array}{l}\text { Average no. } \\
\text { of citations } \\
\text { per year }\end{array}$ & $\begin{array}{l}\text { Altmetric } \\
\text { Attention } \\
\text { Score }\end{array}$ \\
\hline 1. Arthritis Rheum. 2006;54:2665-2673. & 1617 & 108 & 9 \\
\hline 2. Nat Genet. 2009;41:199-204. & 841 & 70 & 30 \\
\hline 3. Lancet. $2005 ; 366: 1367-1374$. & 756 & 47 & 6 \\
\hline 4. Lancet. 2015;386:983-994. & 569 & 95 & 25 \\
\hline 5. Arthritis Rheum. 2009;60:976-986. & 391 & 33 & 13 \\
\hline 6. Arch Dermatol Res. 2011;303:1-10. & 389 & 39 & 33 \\
\hline 7. Ann Rheum Dis. 2009;68:1387-1394. & 345 & 29 & 1 \\
\hline 8. Rheumatology. 2003;42:1460-1468. & 329 & 18 & 13 \\
\hline 9. Arthritis Rheum. 2018;70:345-355. & 302 & 60 & 27 \\
\hline 10. J Am Acad Dermatol. 2017;76:405-417. & 251 & 63 & 509 \\
\hline 11. Arthritis Rheum. 2009;61:233-239. & 249 & 21 & 1 \\
\hline 12. Ann Rheum Dis. 2014;73:48-55. & 234 & 33 & 27 \\
\hline 13. Lancet. 2007;370:272-284. & 223 & 16 & 10 \\
\hline 14. Arthritis Rheum. 2005;52:2513-2518. & $\mathrm{n} / \mathrm{a}$ & 14 & $\mathrm{n} / \mathrm{a}$ \\
\hline 15. Br J Rheumatol. 1994;33:834-839. & 217 & 8 & 3 \\
\hline 16. Am J Clin Dermatol. 2008;9:1-14. & 214 & 16 & 1 \\
\hline 17. J Am Acad Dermatol. 2007;57:1-27 & 207 & 14 & 10 \\
\hline 18. Rheumatology. 2003;42:778-783. & 195 & 11 & $\mathrm{n} / \mathrm{a}$ \\
\hline 19. J Invest Dermatol. 2010;13:1213-1226. & 195 & 17 & $\mathrm{n} / \mathrm{a}$ \\
\hline 20. Dermatology. 1996;193:300-303. & 183 & 7 & $\mathrm{n} / \mathrm{a}$ \\
\hline 21. J Invest Dermatol. 2006;126:740-745. & 182 & 9 & $\mathrm{n} / \mathrm{a}$ \\
\hline 22. Arthritis Care Res (Hoboken). 2011;63(suppl 11):S64-S85. & 139 & 14 & 15 \\
\hline 23. J Invest Dermatol. 2002;118:362-365. & 136 & 7 & 1 \\
\hline 24. J Am Acad Dermatol. 2002;46:867-873. & 131 & 7 & $\mathrm{n} / \mathrm{a}$ \\
\hline 25. J Dermatol. 2012;39:242-252. & 124 & 13 & 3 \\
\hline 26. Br J Dermatol. 2015;173:949-961. & 121 & 20 & 23 \\
\hline 27. J Rheumatol. 2006;33:1417-1421. & 120 & 8 & 1 \\
\hline 28. Rheumatology. 2004;43:790-794. & 113 & 7 & $\mathrm{n} / \mathrm{a}$ \\
\hline 29. J Am Acad Dermatol. 1995;32:78-88. & 113 & 4 & $\mathrm{n} / \mathrm{a}$ \\
\hline 30. Ann Rheum Dis. 2011;70(suppl 1):i77-i84. & 113 & 11 & $\mathrm{n} / \mathrm{a}$ \\
\hline
\end{tabular}




\section{RESEARCH LETTER}

eTABLE. (continued)

\begin{tabular}{|c|c|c|c|}
\hline Reference & $\begin{array}{l}\text { Total } \\
\text { citations }\end{array}$ & $\begin{array}{l}\text { Average no. } \\
\text { of citations } \\
\text { per year }\end{array}$ & $\begin{array}{l}\text { Altmetric } \\
\text { Attention } \\
\text { Score }\end{array}$ \\
\hline 31. Ann Rheum Dis. 2009;68:497-501. & 105 & 9 & 3 \\
\hline 32. J Am Acad Dermatol. 2008;58:224-231. & 105 & 8 & $\mathrm{n} / \mathrm{a}$ \\
\hline 33. Drugs. 2014;74:423-441. & 105 & 15 & 481 \\
\hline 34. J Eur Acad Dermatol Venereol. 2009;23(suppl 1):9-13. & 103 & 8 & 1 \\
\hline 35. J Rheumatol. 2007;34:123-129. & 100 & 7 & 4 \\
\hline 36. Br J Dermatol. 2010;163:580-585. & 99 & 9 & 3 \\
\hline 37. Arch Dermatol. 1969;99:567-579. & 97 & 2 & $\mathrm{n} / \mathrm{a}$ \\
\hline 38. Dermatology. 2009;218:97-102. & 95 & 8 & 2 \\
\hline 39. Ann Rheum Dis. 2010;69:394-399. & 94 & 8 & 3 \\
\hline 40. Arch Dermatol. 2010;146:721-726. & 86 & 8 & $\mathrm{n} / \mathrm{a}$ \\
\hline 41. Int J Dermatol. 2004;43:654-658. & 84 & 5 & $\mathrm{n} / \mathrm{a}$ \\
\hline 42. Ann Rheum Dis. 2012;71:553-556. & 82 & 9 & 4 \\
\hline 43. J Cutan Med Surg. 2003;7:317-321. & 78 & 4 & $\mathrm{n} / \mathrm{a}$ \\
\hline 44. Pediatr Int. 2006;48:525-530. & 78 & 5 & 3 \\
\hline 45. J Rheumatol. 1999;26:1752-1756. & 76 & 3 & 6 \\
\hline 46. Br J Dermatol. 1998;139:655-659. & 70 & 3 & 3 \\
\hline 47. J Ultrasound Med. 2009;28:1569 & 70 & 6 & $\mathrm{n} / \mathrm{a}$ \\
\hline 48. Br J Dermatol. 2014;170:59-65. & 69 & 10 & 1 \\
\hline 49. Dermatology. 2012;225:231-235 & 67 & 7 & 4 \\
\hline 50. Cutis. 2001;68:355-358. & 67 & 3 & 1 \\
\hline 51. Arch Dermatol. 2009;145:269-271. & 67 & 6 & 1 \\
\hline 52. Dermatol Ther. 2007;20:60-67. & 66 & 5 & 3 \\
\hline 53. Br J Dermatol. 2003;148:233-235. & 66 & 4 & $\mathrm{n} / \mathrm{a}$ \\
\hline 54. J Eur Acad Dermatol Venereol. 2009;23(suppl 1):15-21. & 66 & 5 & 23 \\
\hline 55. J Eur Acad Dermatol Venereol. 2009;23:896-904. & 65 & 5 & $\mathrm{n} / \mathrm{a}$ \\
\hline 56. Acta Dermatol Venereol. 2007;87:335-340. & 65 & 5 & $\mathrm{n} / \mathrm{a}$ \\
\hline 57. Arthritis Rheum. 2009;61:1235-1242. & 65 & 5 & $\mathrm{n} / \mathrm{a}$ \\
\hline 58. Arthritis Res Ther. 2010;12:R117. & 64 & 6 & $\mathrm{n} / \mathrm{a}$ \\
\hline 59. Dermatology. 2010;22(suppl 1):1-5. & 63 & 6 & 7 \\
\hline 60. J Rheumatol. 2006;33:1315-1319. & 61 & 4 & $\mathrm{n} / \mathrm{a}$ \\
\hline
\end{tabular}




\begin{tabular}{|c|c|c|c|}
\hline Reference & $\begin{array}{l}\text { Total } \\
\text { citations }\end{array}$ & $\begin{array}{l}\text { Average no. } \\
\text { of citations } \\
\text { per year }\end{array}$ & $\begin{array}{l}\text { Altmetric } \\
\text { Attention } \\
\text { Score }\end{array}$ \\
\hline 61. Clin Rheumatol. 2000;19:301-305. & 61 & 3 & $\mathrm{n} / \mathrm{a}$ \\
\hline 62. J Am Acad Dermatol. 1991;24:598-602. & 60 & 2 & $\mathrm{n} / \mathrm{a}$ \\
\hline 63. J Eur Acad Dermatol Venereol. 2011;25(suppl 2):19-27. & 58 & 6 & 1 \\
\hline 64. J Dermatol. 2017;44:355-362. & 57 & 14 & $\mathrm{n} / \mathrm{a}$ \\
\hline 65. Clin Dermatol. 2008;26:448-459. & 55 & 4 & $\mathrm{n} / \mathrm{a}$ \\
\hline 66. Arch Dermatol. 2011;147:429-436. & 55 & 5 & 1 \\
\hline 67. Br J Dermatol. 2014;171:1123-1128. & 54 & 8 & 3 \\
\hline 68. Br J Dermatol. 2008;159:453-456. & 53 & 4 & 3 \\
\hline 69. Int J Dermatol. 2002;41:220-224. & 53 & 3 & $n / a$ \\
\hline 70. Semin Cutan Med Surg. 2009;28:39-43. & 52 & 2 & $\mathrm{n} / \mathrm{a}$ \\
\hline 71. Cutis. 1999;64:309-314. & 52 & 2 & $n / a$ \\
\hline 72. Arthritis Rheum. 2016;68:915-923. & 52 & 10 & 74 \\
\hline 73. J Am Acad Dermatol. 2016;74:134-142. & 51 & 10 & 10 \\
\hline 74. J Dtsch Dermatol Ges. 2014;12:48-57. & 51 & 7 & $\mathrm{n} / \mathrm{a}$ \\
\hline 75. Cutis. 1992;50:174-178. & 51 & 2 & $\mathrm{n} / \mathrm{a}$ \\
\hline 76. Arthritis Rheum. 2012;64:3150-3155. & 51 & 6 & 12 \\
\hline 77. J Rheumatol. 2006;33:1452-1456. & 50 & 3 & $\mathrm{n} / \mathrm{a}$ \\
\hline 78. Lancet. 2018;391:2273-2284. & 50 & 16 & 149 \\
\hline 79. J Eur Acad Dermatol Venereol. 2011;25:1409-1414. & 49 & 5 & $n / a$ \\
\hline 80. Br J Dermatol. 2000;142:1171-1176. & 49 & 2 & $\mathrm{n} / \mathrm{a}$ \\
\hline 81. Rheum Dis Clin North Am. 2015;41:569-579. & 49 & 8 & 1 \\
\hline 82. Pediatr Dermatol. 2013;30:424-428. & 48 & 6 & 1 \\
\hline 83. Am Fam Physician. 2013;87:626-633. & 48 & 6 & 3 \\
\hline 84. Best Pract Res Clin Rheumatol. 2012;26:805-822. & 48 & 5 & $\mathrm{n} / \mathrm{a}$ \\
\hline 85. J Eur Acad Dermatol Venereol. 2011;25:1080-1084. & 48 & 5 & 1 \\
\hline 86. Ann Rheum Dis. 2011;70(suppl 1):i71-i76. & 48 & 5 & 1 \\
\hline 87. Postgrad Med. 2009;121:154-161. & 48 & 4 & $\mathrm{n} / \mathrm{a}$ \\
\hline 88. Dermatology. 2003;206:153-156. & 48 & 3 & 6 \\
\hline 89. J Eur Acad Dermatol Venereol. 2011;25:579-586. & 48 & 5 & $\mathrm{n} / \mathrm{a}$ \\
\hline 90. Curr Opin Rheumatol. 2009;21:340-347. & 47 & 4 & $\mathrm{n} / \mathrm{a}$ \\
\hline
\end{tabular}




\section{RESEARCH LETTER}

eTABLE. (continued)

\begin{tabular}{|c|c|c|c|}
\hline Reference & $\begin{array}{l}\text { Total } \\
\text { citations }\end{array}$ & $\begin{array}{l}\text { Average no. } \\
\text { of citations } \\
\text { per year }\end{array}$ & $\begin{array}{l}\text { Altmetric } \\
\text { Attention } \\
\text { Score }\end{array}$ \\
\hline 91. Med Res Rev. 2014;34:438-454. & 46 & 7 & 2 \\
\hline 92. Rheumatol Ther. 2016;3:91-102. & 45 & 9 & 13 \\
\hline 93. J Dermatol. 2012;39:253-259. & 45 & 5 & $\mathrm{n} / \mathrm{a}$ \\
\hline 94. J Eur Acad Dermatol Venereol. 2010;24:530-534. & 45 & 4 & $\mathrm{n} / \mathrm{a}$ \\
\hline 95. Nat Rev Rheumatol. 2014;10:531-542. & 44 & 6 & 2 \\
\hline 96. Br J Dermatol. 2005;153:1153-1158. & 44 & 3 & $\mathrm{n} / \mathrm{a}$ \\
\hline 97. Br J Dermatol. 2003;149:207-209. & 44 & 2 & $\mathrm{n} / \mathrm{a}$ \\
\hline 98. J Am Acad Dermatol. 2005;52:736-737. & 43 & 3 & $\mathrm{n} / \mathrm{a}$ \\
\hline 99. Dermatology. 1999;199:313-318. & 43 & 2 & 3 \\
\hline 100. Br J Dermatol. 2014;170:398-407. & 42 & 6 & 4 \\
\hline
\end{tabular}

Abbreviation: $\mathrm{n} / \mathrm{a}$, not available. 